\title{
Pore Characterization of Alumina Short Fiber Using Small Angle X-Ray Scattering Technique
}

\author{
Umar Abdillah Anwar ${ }^{1}$, Hafizal Yazid ${ }^{2 *}$, Ruey Shan Chen $^{1}$, Siti Zaubidah ${ }^{1}$, Nurulizzati Makhtar ${ }^{1}$, \\ Maria Sabtu $^{1}$, M Rawi M Zain ${ }^{2}$, Roslan Yahya ${ }^{3}$ and Sahrim Ahmad ${ }^{1}$ \\ ${ }^{1}$ Department of Applied Physics, Universiti Kebangssan Malaysia, Malaysia \\ ${ }^{2}$ Materials Technology Group, Malaysia \\ ${ }^{3}$ Plant Assessment Technology Group, Malaysia
}

*Corresponding author: Hafizal Yazid, Materials Technology Group, Industrial Technology Division, Malaysian Nuclear Agency, 43000 Kajang Selangor, Malaysia

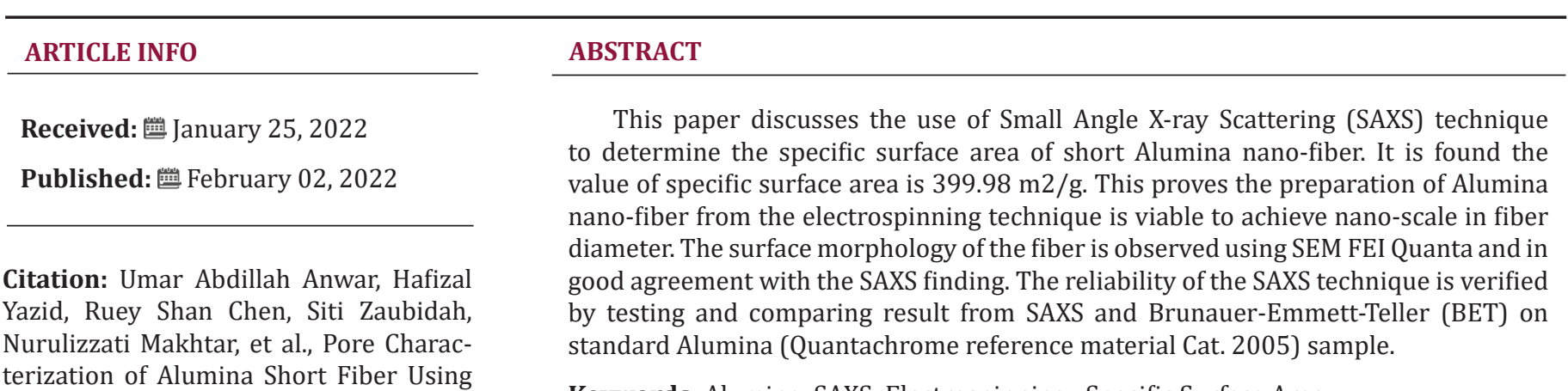

terization of Alumina Short Fiber Using Small Angle X-Ray Scattering Technique. Biomed J Sci \& Tech Res 41(4)-2022. BJSTR. MS.ID.006622.
Keywords: Alumina; SAXS; Electrospinning ; Specific Surface Area

\section{Introduction}

Alumina or Aluminium Oxide ( $\mathrm{Al} 2 \mathrm{O} 3$ ) is ceramic metal oxide and have wide variety of applications [1]. Alumina is also widely used as biomaterials for bone replacement [2]. This is due to the fact that Alumina is relatively high strength and has good tribological properties especially wear resistance. Improvement in terms of material purity has led to great chemical resistivity and excellent tissue compatibility. Thus, it is not surprising the usage of Alumina even is found in dental implants [3]. Alumina could be produced by electrospinning technique. It is a process of producing fibers by employing electrohydrodynamic properties with a relatively simple set-up [4]. The set-up includes a high voltage power supply, pump, syringe and grounded collector. Raw material for the electrospinning process is in the form of sol-gel. Prior to sol-gel injection by using the syringe, an electrical field is generated between the syringe and the collector. As the sol-gel is injected from the syringe, an electrical repulsive force directs the material in the form of fiber onto the collector. The obtained material on the collector finally in the form of thin layer of mats. The material is carefully removed and calcined at high temperatures to remove the unwanted polymer precursor. Then the desired Alumina is obtained. The fiber produced by this technique demonstrate good performance and properties. This is due to the increase of the specific surface area of the nanofiber. The nano-scale could be achieved by various approaches includes reduction of fiber diameter. Electrospinning process allows manipulation of setting parameters to achieve that [5]. Electron microscopy (EM) and small angle scattering (SAXS) are two approaches of data acquisition method for characterizing nanoparticles. Both are considered as direct technique but one is in real space (EM) and the other is in reciprocal space (SAXS). 
These two methods are usually utilized to produce data such as size distributions, determine pore sizes, high resolution imaging and much more [6]. Fundamentally, electron microscopy (EM) uses high speed electron as a source of illumination to obtain high resolution image while small angle x-ray scattering (SAXS) involves successive irradiation mode of passing the $\mathrm{x}$-ray beam through the sample and data built based on scattered $\mathrm{x}$-rays $[7,8]$.

There are idiosyncratic advantages and disadvantages between these two techniques. With regard to application on different types of samples, small angle x-ray scattering (SAXS) has further advantages due being able to study variety of sample states includes solid, powder, sol-gel as well as thin film [9]. Estimation of particle sizes of electron microscopy (EM) methods is made from measurements of limited number of particles which makes small angle $\mathrm{x}$-ray scattering (SAXS) provide more accurate data on particle sizes due the estimation is made from vast number of particles [10]. In addition, preparing samples for small angle x-ray scattering (SAXS) are often require very little time, comparing to the complicated electron microscopy (EM) sample preparation procedure where often taking longer period. It is the interest of researchers to probe the specific surface area for confirming the success of obtaining nano-scale materials. One of the technique is through the use of Small Angle X-ray Scattering (SAXS) technique.

SAXS is advantageous as compared to fluid for probing the pore space. It covers both close and open pore. In SAXS, a beam of $\mathrm{X}$-rays of fixed wavelength and known intensity is illuminated on the sample, and the intensity of the scattered signal is measured versus the scattering vector. X-ray probe the fluctuations of the electronic density and reflect the chemical heterogeneity and density variations within the sample. From this result, many information on the sample are obtainable such as size, shape and specific surface area.

\section{Materials and Methods}

The Alumina nanofibers were prepared from electrospinning process. The raw materials used were reagent grade Aluminium Isopropoxide (AIP) as the source of alumina precursor, Polyvinylpyrrolidone (PVP with Mw 1300000) as polymer precursor, nitric acid (HNO3) and Ethyl Acetoacetate. All chemicals were from Sigma Aldrich. The optimum electrospinning setting parameter were followed based on previous work [11]. Surface morphology was carried out using SEM/EDX FEI Quanta 400. SAXS experiments were performed using Anton Paar Saxpoint instrument, operated at $50 \mathrm{kV}$ and $1 \mathrm{~mA}$ with the point collimation geometry. The radiation used was a $\mathrm{Ni}$ filtered $\mathrm{CuK} \alpha$ radiation of wavelength $0.154 \mathrm{~nm}$. The intensity profiles are recorded using a CMOS 2-D detector (Eiger) and the scattering vector $\mathrm{q}$, covers from 0.035 to $5 \mathrm{~nm}-1$. One-dimensional scans of I(q) were extracted from two- dimensional scattering patterns using the analysis package SAXS analysis, Primus and Easyswax. Angular calibration of the scattered intensities in the small angle regime for the detector is performed using silver bahenate $(d=58.38 \AA$ ). Sample to detector distance is $575.5 \mathrm{~mm}$ and sample exposure to the source is 600 seconds. All experiments are carried out at room temperature, $20^{\circ} \mathrm{C}$. The measured scattering vector is related to the scattering angle through the equation:

$$
q=\frac{4 \pi}{\lambda} \sin \theta
$$

where:

$\theta=$ Scattering angle or half of diffraction angle (degree)

$\mathrm{q}=$ scattering vector $(\mathrm{nm}-1)$

$\lambda=0.154(\mathrm{~nm})$

Another parameter under consideration is Invariant. It is the integral of second moment of the scattering curve I(q) $\mathrm{q}^{2}$. The equation is given below:

$$
Q_{p}=\int_{0}^{\infty} q^{2} \Delta I(q) d q\left(\mathrm{a} . \mathrm{u} / \mathrm{nm}^{3}\right)
$$

Qp shall be calculated by extrapolating scattering intensity towards zero (Guinier approximation) and infinitely large scattering angle (Porod's q-4 dependence). Pore is assumed to be spherical in shape.

Surface to volume ratio is based on the equation of:

$$
\frac{s}{v}=1000 \pi \frac{K p}{Q p} \theta(1-\theta)\left(\mathrm{m}^{2} / \mathrm{cm}^{3}\right)
$$

where:

$$
\begin{aligned}
& \mathrm{Kp}=\text { Porod Constant } \\
& \mathrm{Qp}=\text { Invariant } \\
& \theta=\text { Volume fraction of particles }
\end{aligned}
$$

Specific surface is obtained by dividing the surface to volume ratio with the apparent density. All equations are based on Heimo Schnablegger [12]. SAXS experiment were subjected to two samples namely Alumina Quantachrome Reference standard and Alumina nanofiber. The result for Alumina Quantachrome Reference standard is compared with the value given by the manufacturer but by using a different technique, BET technique. Then, the second sample, Alumina nanofiber is subjected to SAXS measurement. 2D-SAXS image of Alumina Quantachrome Reference (Figure 1) and Alumina nanofiber (Figure 2) with the same linear scaling in the q-range of $0.035 \mathrm{~nm}^{-1}<\mathrm{q}_{\mathrm{z}}<5 \mathrm{~nm}^{-1}$. Exposure time was 300s (avarage of 2 reading). 2D scattering data are reduced to $1 \mathrm{D}$ scattering 
profile by radial integration from $0^{\circ}-180^{\circ}$. Further analysis is performed based on 1D scattering profile as shown in (Figure 3). From Invariant (Porod and Guinier) calculation, It was found the value of Kp/Qp are 0.19 for standard Alumina Quantachrome Reference sample and 0.62 for Alumina sintered nanofiber sample.
The specific surface area for both sample is calculated and given in (Table 1). The existance of nanopores are clearly visible by the excess SAXS signal in (Figures 1 \& 2). Ring pattern for both sample indicates there is no preferred orientation of the nanopore within the plane of the samples.

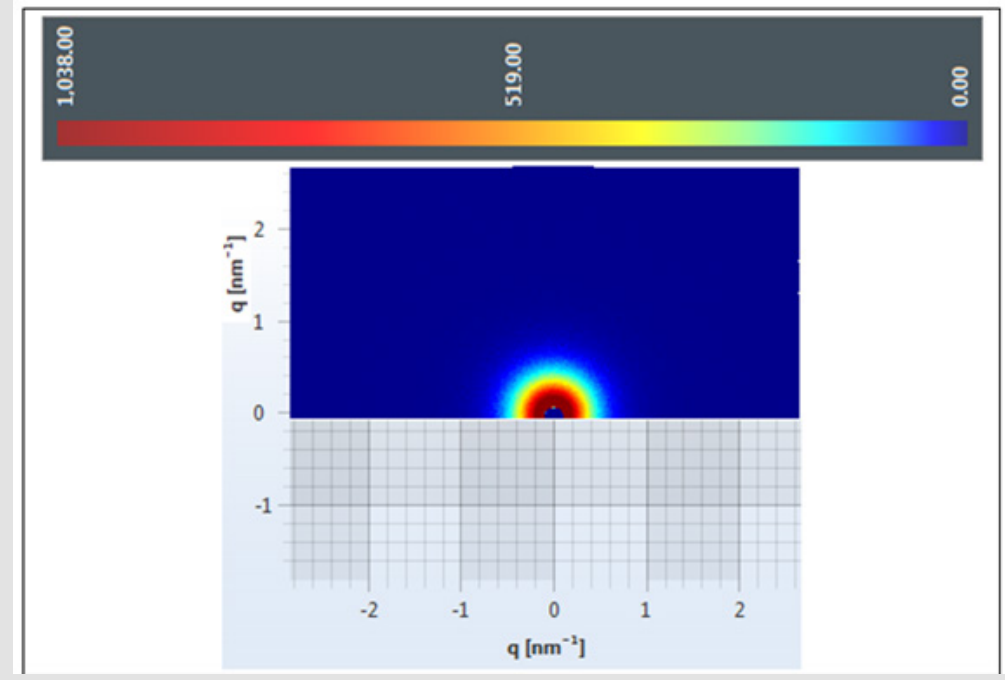

Figure 1: 2D scattering of Alumina Quantachrome Reference sample.

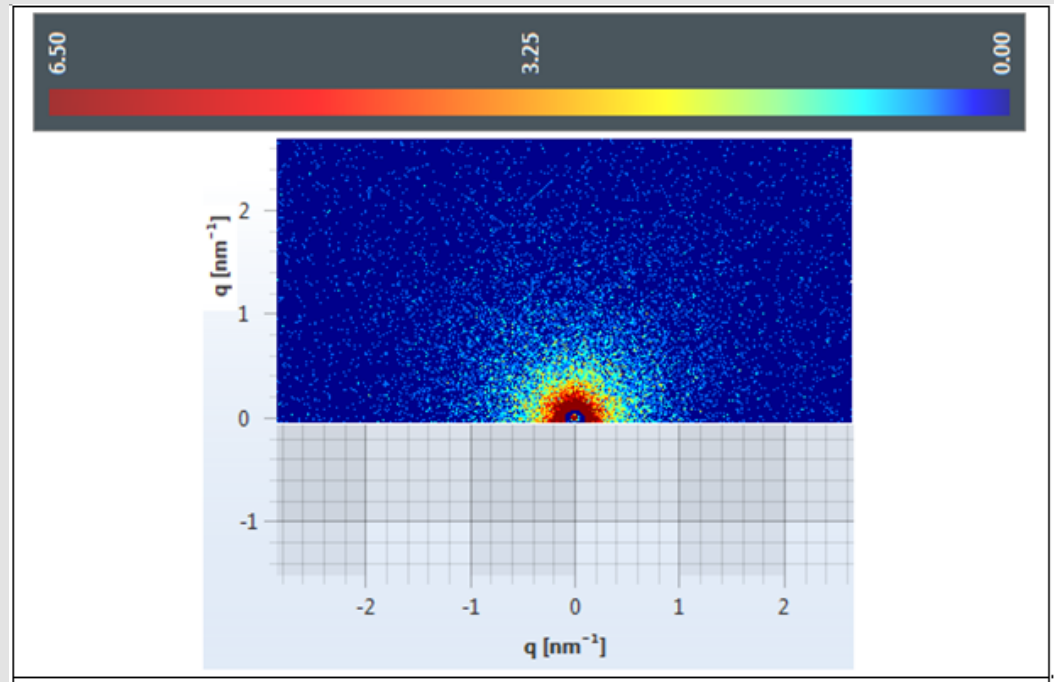

Figure 2: 2D scattering of Alumina nanofiber sample.

Table 1: Specific surface area.

\begin{tabular}{|c|c|c|}
\hline Sample & Specific surface area $(\mathbf{m} 2 / \mathbf{g})$, SAXS & $\begin{array}{c}\text { Specific surface area (m } \mathbf{2} / \mathbf{g}), \text { BET. The value is } \\
\text { from manufacturer. }\end{array}$ \\
\hline Alumina Quantachrome Reference standard & 95.58 & 107.5 \\
\hline Alumina sintered nanofiber & 399.98 & - \\
\hline
\end{tabular}


The intensity of alumina Quantachrome Reference standard is found much more higher than the Alumina sintered nanofiber. This reflect that many scatterer in the alumina Quantachrome Reference sample as intended from the reference material. In terms of pore size, it is found that the alumina nanofiber exhibits smaller pore size as indicated by the higher specific surface area, $399.98 \mathrm{~m}^{2} / \mathrm{g}$. Comparing specific surface area values for alumina Quantachrome Reference material between SAXS and BET technique reveals that both values are in good agreement. SAXS gives the value of $95.58 \mathrm{~m}^{2} / \mathrm{g}$ and BET is reported to give the value of $107.5 \mathrm{~m} 2 / \mathrm{g}$. However, slight differences might be caused by damages to the sample during the crushing of the Quantachrome Reference sample from solid to powder form in order to mount the sample into the holder for SAXS experiment. The surface morphology of Alumina sintered nanofiber is given in (Figure 4). it is clearly seen the fibers are in the range of $100-200 \mathrm{~nm}$ in diameter. The pore between the fibers are expected to be much more smaller than that. This will give rise to the scattering event in the sample. The applicability of SAXS technique in assessing the pore in the sample is found to be comparable to BET technique. The simple sample preparation makes the technique very attractive and is successfully shown in this work by probing Alumina sintered nanofiber.

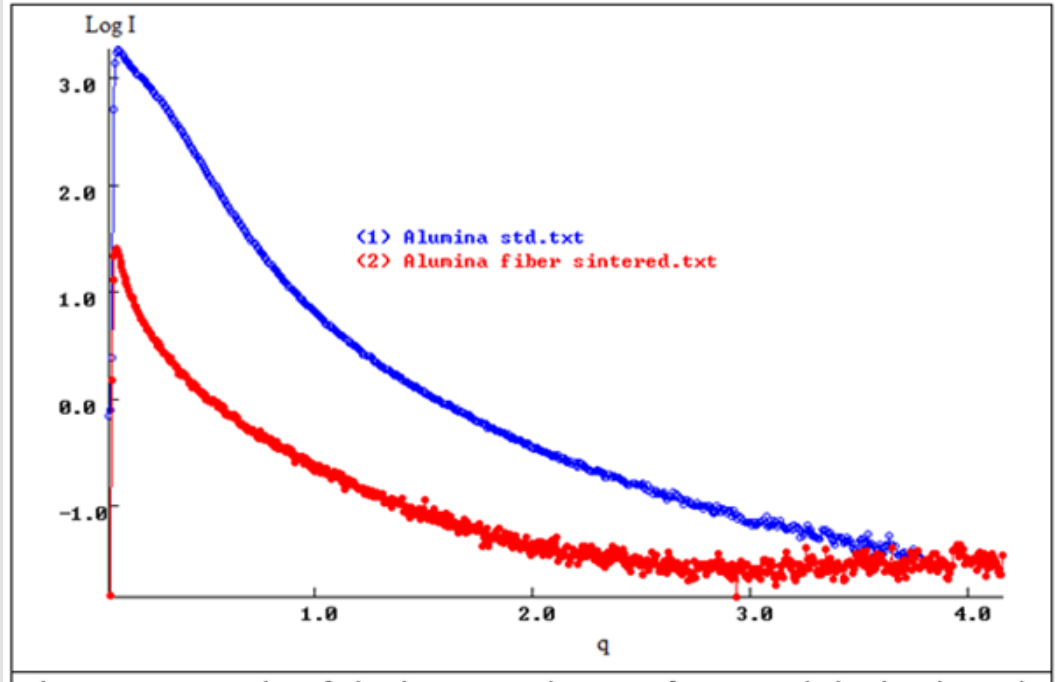

Figure 3: 1D scattering of Alumina Quantachrome Reference and Alumina sintered nanofiber sample.

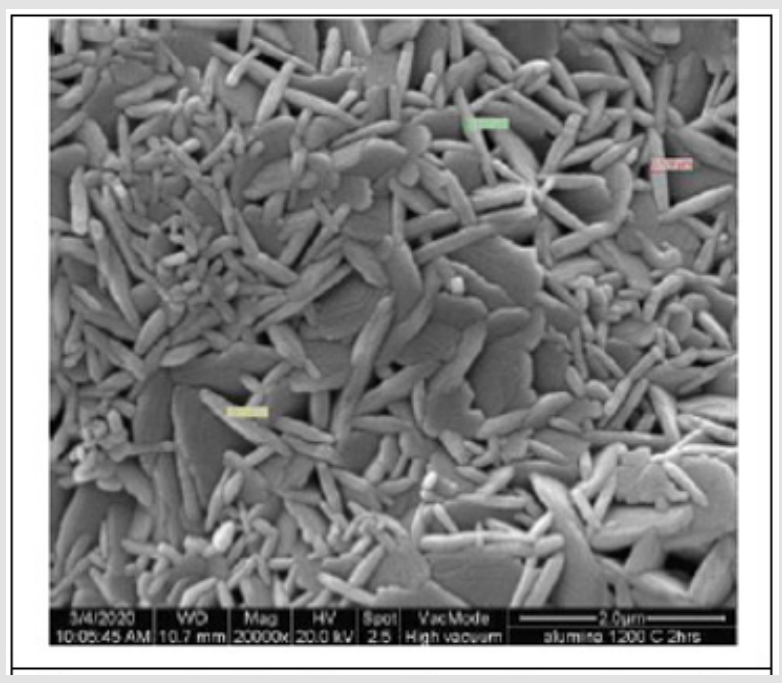

Figure 4: Alumina sintered nanofiber, short fiber with the average diameter of 100- $200 \mathrm{~nm}$.

\section{Acknowledgement}

The authors would like to thank the Nuclear Malaysia and Ministry of Higher Education (MOHE) for the research financial support under the research scheme of Fundamental Research Grant (FRGS) with the grant number FRGS/1/2018/STG07/ MOSTI/02/01. 


\section{References}

1. Seyed Masoud Emarati, MahdiMozammel (2020) Efficient one-step fabrication ofsuperhydrophobic nano-TiO2/TMPSi ceramic composite coating with enhanced corrosionresistance on 316L. Ceramics Int 46(2): 1652-1661.

2. Maryam Rahmati, Masoud Mozafari (2019) Biocompatibility of aluminabased biomaterials-A review. J Cell Physiol 234(4): 3321-3335.

3. VasanthGopal, GeethaManivasagam (2019) Zirconia-alumina composite for orthopedic implant application. Woodhead Pub Ser in Biomat, pp. 201-219.

4. Dan Li, Jesse, T McCann, Younan Xia, Manuel Marquez (2006) Electrospinning: A simple and versatile technique for producing ceramic nanofibers and nanotubes. J of the American Ceramic Soc 89(6): 18611869.

5. GibinGeorge, T Senthil, ZhipingLuo, S Anandhan (2021) Sol-gel electrospinning of diverse ceramic nanofibers and their potential applications. Woodhead Pub Ser in Comp Sci and Eng (2021): 689-764

6. Pablo Hernandez Cerdan, Bradley W Mansel, Andrew Leis, Leif Lundin, Martin AK Williams (2020) Structural analysis of polysaccharide networks by transmission electron microscopy: comparison with smallangle x-ray scattering. Biomacromolecules 19(3): 989-995.

ISSN: 2574-1241

DOI: 10.26717/BJSTR.2022.41.006622

Hafizal Yazid. Biomed J Sci \& Tech Res

(C) (P) This work is licensed under Creative

Submission Link: https://biomedres.us/submit-manuscript.php
7. Qiaoli Chen, Christian Dwyer, Guan Sheng, Chongzhi Zhu, Xiaonian Li, et al. (2020) Imaging Beam-Sensitive Materials by Electron Microscopy. Advanced Materials 32: 16.

8. Kun Qian, Randall E Winans, Tao Li (2021) Insights into the nanostructure, solvation, and dynamics of liquid electrolytes through small-angle x-ray scattering. Advanced Energy Materials 11: 4.

9. Lauren Boldon, Fallon Laliberte, Li Liu (2015) Review of the fundamental theories behind small angle X-ray scattering, molecular dynamics simulations, and relevant integrated application. Nano Review 6: 1.

10. Maxim V Petoukhov, Dmitri I Svergun (2013) Review: Applications of small-angle X-ray scattering to biomacromolecular solutions. 45(2): 429-437.

11. Umar Abdillah, Hafizal Yazid, Sahrim Ahmad, Nurulizzati Makhtar, Siti, et al. (2021) The effect of various electrospinning parameter on preparation of alumina nanofibers. IOP Conf Ser Mater Sci Eng, pp. 1106.

12. Heimo Schnablegger, Yashveer Singh (2011) The SAXS Guide: Getting acquainted with the principles Anton Paar GmbH Austria.

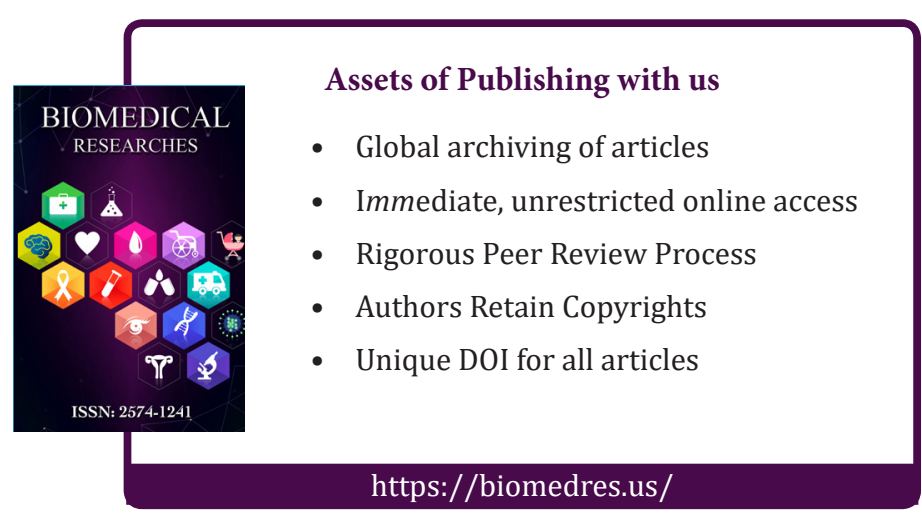

